\title{
A near field 3D radar imaging technique
}

\section{Anguela, A. Broquetas}

Dept. Teoria del Senyal i Comunicacions, Grup AMR

ETSE Telecomunicació - UPC P.O. Box 30002, 08080

Barcelona, Spain Tel. +3434017221 , Fax +3434017232

\section{Abstract}

The paper presents an algorithm which recovers a $3 \mathrm{D}$ reflectivity image of a target from near-field scattering measurements. Spherical wave nearfield illumination is used, in order to avoid a costly compact range installation to produce a plane wave illumination. The system is described and some simulated 3D reconstructions are included. The paper also presents a first experimental validation of this technique.

\section{Introduction}

Processing the coherent radar cross section (RCS) data scattered by a target it is possible to reconstruct its reflectivity image by inverse synthetic aperture radar (ISAR) techniques [Mensa(l)].

Measurements of RCS require a plane wave illumination of the target. This wave-front can be obtained working in far field conditions or using a compact range based on a parabolic reflector [Broquetas et al(2)]. Both methods lead to a costly installation. Moreover, a compact range involves some inherent technical problems (edge diffraction, surface tolerances, etc.).

The paper presents an imaging algorithm, which reconstructs the 3D reflectivity of the target from backscattering measurements using spherical wave fronts which are easily produced by low directivity simple antennas like horns. The formulation is based on the $2 \mathrm{D}$ spherical wave ISAR technique [Broquetas et al(3)], adding a vertical scan of the antennas to the usual target azimuth rotation and frequency sweep.

\section{Formulation}

The system layout is shown in Fig. 1. The target is illuminated with a spherical wave. Measurements of the reflected wave are made as a function of 3 variables: the object rotation $\theta$, the antenna displacement $h$ and the operating frequency sweep $f$, giving a 3D hologram. Initially a monostatic geometry with an unique isotropic antenna is assumed, bistatic condition and the radiation diagram of the antennas is included later.

The target can be considered to be formed by a number of independent point scatterers distribution and, consequently, can be described by means of a $3 \mathrm{D}$ spatial reflectivity function $\psi(x, y, z)$. Our objective is to obtain a good approximation of this function by applying a focusing operator $\xi$ on the scattered field $\operatorname{Es}(f, \theta, h)$, as indicated in the following equation

$\Psi^{\prime}(\rho, \varphi, z)=\iint_{f} \int_{h} C E_{s}(f, \theta, h) \xi(f, \theta, h ; \rho, \varphi, z) d f d \theta d h \approx \Psi(\rho, \varphi, z)$

where $\psi^{b}$ is the image of the actual reflectivity $\psi$ expressed in cylindrical coordinates and $\mathrm{C}$ is a constant removed by calibration.

The focusing operator adopted attempts to compensate the amplitude and phase variations along wave propagation path. So the proposed solution is

$$
\xi(f, \theta, h ; \rho, \varphi, z)=e^{j \frac{4 \pi}{l} R} R^{2} f
$$

where $\mathrm{R}$ is the distance between the antenna and every scatter point in the target, as shown in figure 4 , and takes the form

$$
R=\sqrt{D^{2}+\rho^{2}+(z-h)^{2}-2 \rho D \cos (\theta-\varphi)}
$$

If a bistatic geometry is employed, with two antennas separated an angle $\beta$, the focusing operator adopts the form

$$
\xi(f, \theta, h ; \rho, \varphi, z)=e^{j \frac{2 \pi}{\lambda}\left(R_{1}+R_{2}\right)} R_{1} R_{2} f
$$

where

$$
\begin{aligned}
& R_{1}=\sqrt{D_{1}^{2}+\rho^{2}+(z-h)^{2}-2 \rho D_{1} \cos (\theta-\varphi)} \\
& R_{2}=\sqrt{D_{2}^{2}+\rho^{2}+(z-h)^{2}-2 \rho D_{2} \cos (\theta-\varphi+\beta)}
\end{aligned}
$$

Finally, the antennas radiation diagrams have been included in the formulation as a multiplicative factor in the focusing operator $\zeta$. Azimuth and elevation planes have been considered, in amplitude and phase.

\section{Implementation}

Noting that $\zeta(f, \theta, h, \rho, \varphi, z) \equiv \zeta(f, \rho, \theta-\varphi, z-h)$, equation (1) reduces to two-dimensional convolution and can be implemented as a product of Fourier Transforms using FFT's, which drastically reduces the processing time.

$$
\Psi^{\prime}(\rho, \varphi, z)=F F T^{-1}\left\{\sum_{f} F F T_{\varphi, z}\left[E_{s}(f, \varphi, z)\right] F F T_{\varphi, z}[\xi(f, \rho, \varphi, z)]\right\}
$$

Nevertheless, the amount of data of both the hologram and image is very large (tens of MBytes) and the computational cost is very high - a CONVEX C3400 parallel-vectorial supercomputer has been used in this work with a CPU processing time in the order of few hours.

For real measurements, two small horns have been used and their directivity diagrams have been previously characterized. Achieving a high z-resolution is seriously difficulted by the antennas elevation beamwidth, which limits the vertical scan angle.

\section{Results}

Numerical simulations have been carried out in order to insure a proper operation of the algorithm. Fig. 2 shows a reconstructed image of a 3D structure formed by 7 scatterers with relative reflectivity values of 2 (bottom $z$-level) $1,2,4,2$ ( $2^{\text {nd }} z$-level), 2 ( $3^{\text {rd }}$ $\mathrm{z}$-level) and 4 ( $4^{\text {th }} \mathrm{z}$-level). Measurements have been simulated for a frequency range from 18 to $26 \mathrm{GHz}$, azimuth scan of $360^{\circ}$ and vertical or elevation scan of $44^{\circ}$. The reconstructed area is a volume of $20 \times 20 \times 30 \mathrm{~cm}$, with the antennas situated $50 \mathrm{~cm}$ from the Origin.

Fig. 3 shows the reconstruction of 5 scatterers with reflectivity value 1 , aligned along $\mathrm{z}$-axis, for $12-26 \mathrm{GHz}, 360^{\circ}$ azimuth, $44^{\circ}$ elevation and antennas $1 \mathrm{~m}$ away. The quasi-invariance of the point spread function, as well as the uniform sensitivity can be observed. Besides, it has been noticed that vertical resolution can be easily improved by increasing the elevation scan angle.

Finally, the technique has been validated with several measurements in anechoic chamber. Usual calibration techniques have been carried out, similar to 2D SWISAR [Broquetas et al(3)]. The reflectivity image of an helicopter model (see Fig. 7.) has been reconstructed, backscattering data has been obtained with a $8 \mathrm{GHz}$ bandwidth $(18-26 \mathrm{GHz}), 360^{\circ}$ azimuth rotation and $25.7^{\circ}$ elevation (48 cm vertical scan at $105 \mathrm{~cm}$ ), using $\mathrm{HH}$ polarization. Fig. 5 shows five horizontal cuts separated $1.6 \mathrm{~cm}$ each one, where helicopter blades can be clearly resolved from its body. Two orthogonal vertical projections are also shown, which have been obtained incoherently adding vertical cuts of the $3 \mathrm{D}$ reflectivity image. Fig. 6 shows both the transversal projection and the 
longitudinal projection (showing the same view as in Fig. 7). The measurement time is considerably high (50 hours for this example) due to the large amount of information required.

\section{Conclusions}

A 3D imaging algorithm capable of reconstructing volumetric structures has been proposed. Resolution and dynamic range that have been obtained are similar to the conventional plane wave $2 \mathrm{D}$ ISAR reconstruction case. Software implementation and formulation are more complicated and longer CPU time is required in data processing, but the need of costly compact range equipment is avoided. Present work is addressed towards obtaining the target RCS from the date set acquired.

\section{Acknowledgements}

This work has been supported by the Spanish Science and Technology Commission (CICYT TIC 88-288E).

\section{References}

[1] D.L. Mensa, "High resolution radar imaging", 1984, Artech House, Dedham.

[2] A. Broquetas, X. Fàbregas, J. Xarrié, L. Jofre, A. Cardama, "A Compact system for RCS measurements and imaging up to 40 GHz", Nice 1990, Proceedings de Journeés Internationales de Nice sur les Antennes (JINA-90), pp. 596-599.

[3] A. Broquetas, L. Jofre, A. Cardama, "A near field Spherical Wave Inverse Synthetic Aperture Radar technique", Chicago 1992, IEEE Antennas and Propagation Society International Symposium, Volume Two, pp. 114-117

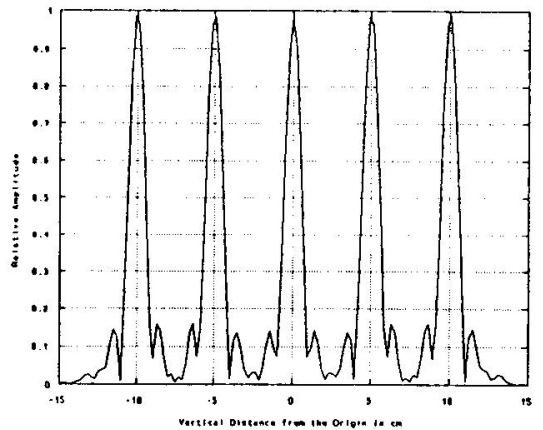

Fig.3 Five scatterers along $\mathrm{z}$-axis
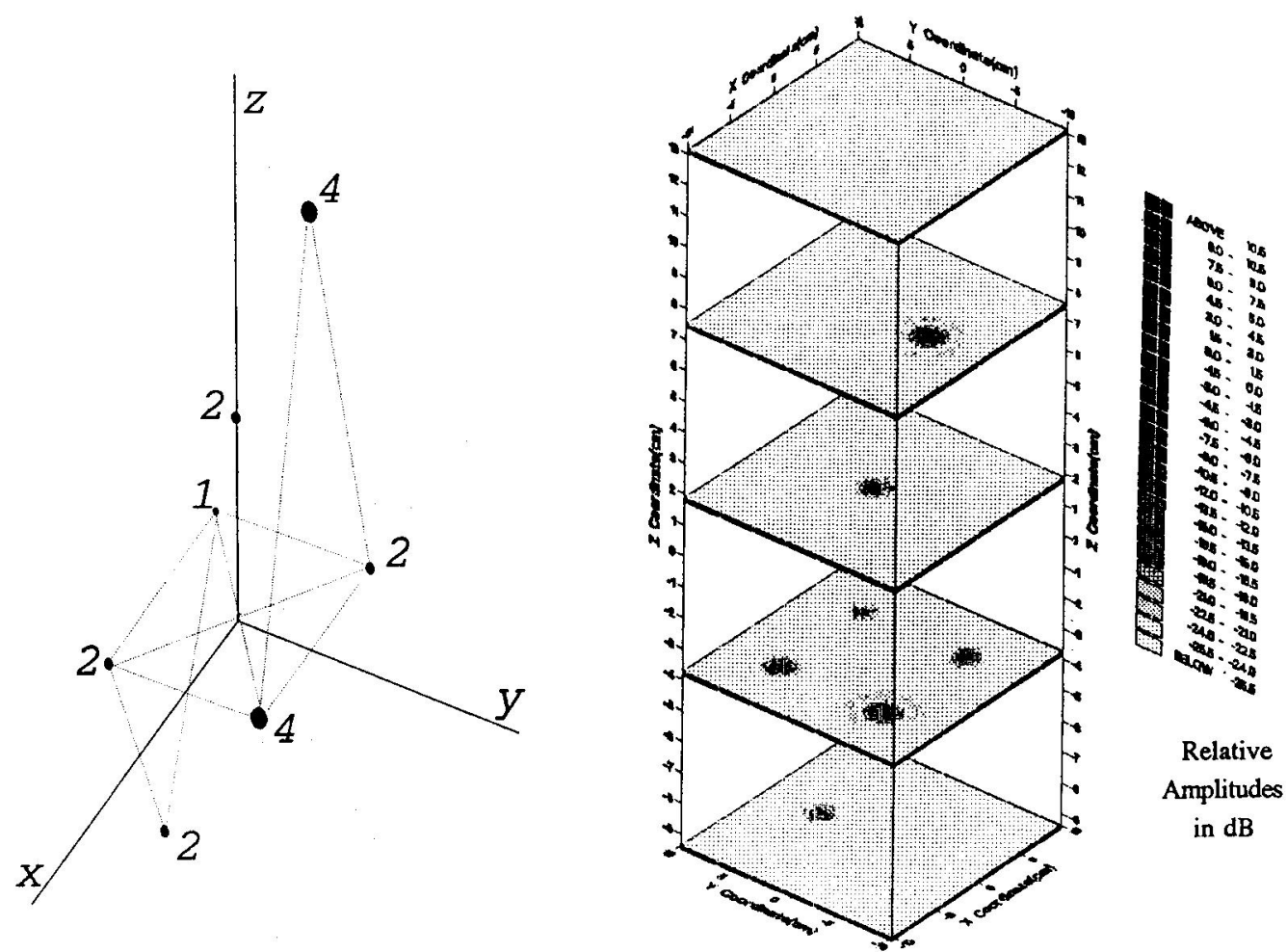

Fig.2. left : 3D scatterers structure - the number indicates relative reflectivity

right: 3D reconstruction of reflectivity $\Psi(x, y, z)$ of the structure. Only five slices (at constant $\mathrm{z}$ level) are shown, the lower four corresponding to the levels where the scatterers are located 

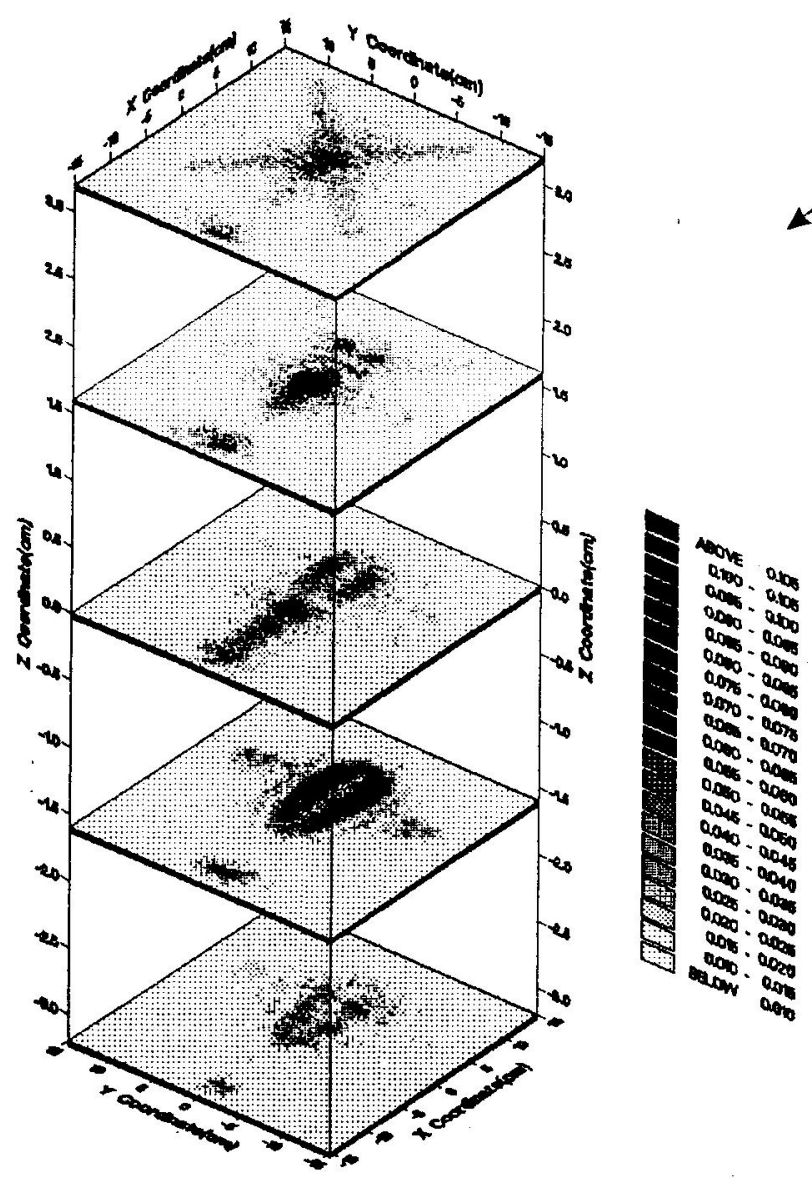

Fig. 5. 3D reconstruction of the helicopter reflectivity, where five horizontal slices equally-spaced are shown (lineal scale)

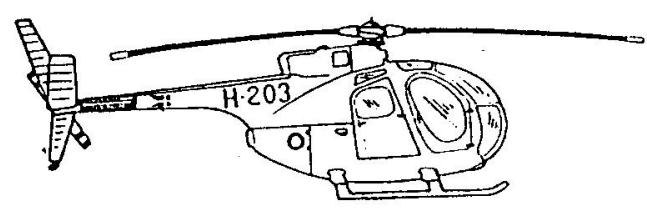

Fig. 7. Hughes $500 \mathrm{C}$ helicopter, 1:32 scale $8 \mathrm{~cm}$ height, $24.5 \mathrm{~cm}$ width (including blades)

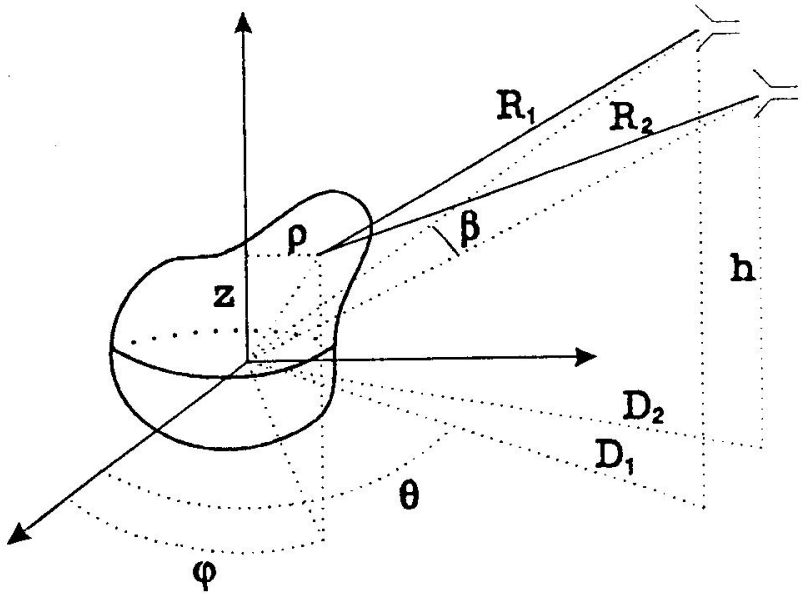

Fig. 4. System Geometry
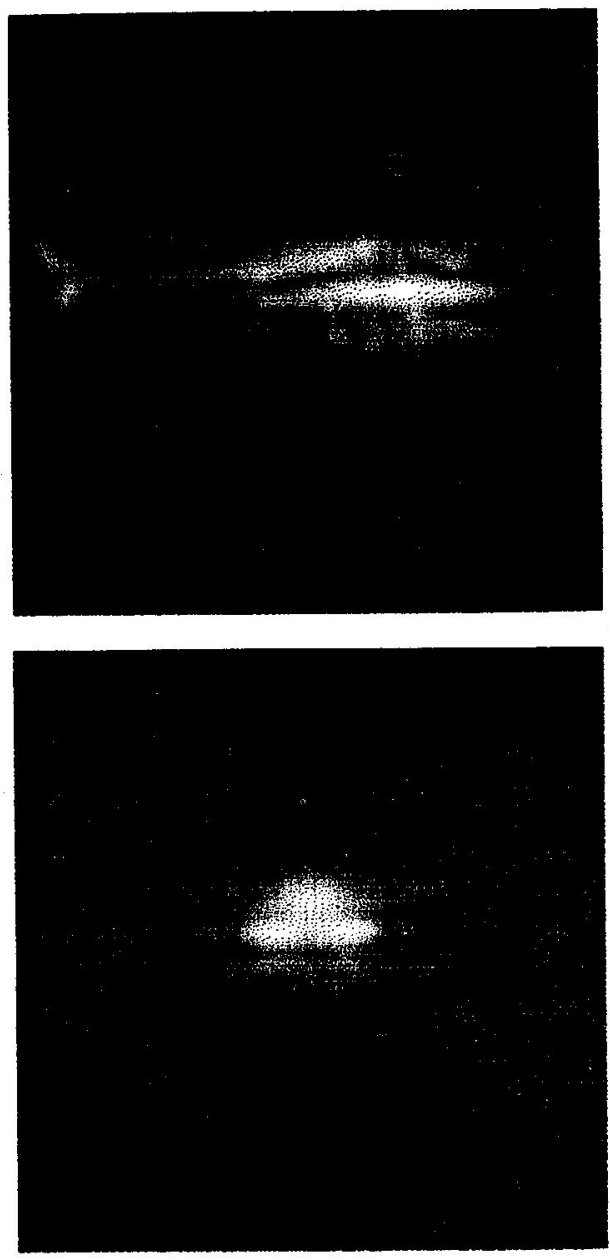

Fig. 6. Vertical projections - longitudinal (up) and transversal (down) showing relative amplitudes in $\mathrm{dB}$ 